Patrik Beno - Miroslav Gutten - Milan Simko - Jozef Sedo*

\title{
STABILIZATION AND CONTROL OF SINGLE-WHEELED VEHICLE WITH BLDC MOTOR
}

To stabilize a single-wheel vehicle is necessary to know the angle of gradient of the platform with respect to the horizontal axis and also the movement of the vehicle. This can be detected in several ways. Accelerometers and gyroscopes are small, accurate and energy-saving today. They provide analogue or digital output. In the introduction we will describe the basic parameters of accelerometer and gyroscope. We will describe the principle of digital signal processing from both sensors. In the next section we look at the effect of dynamic acceleration on the sensors and the elimination of this phenomenon with a complementary filter. We explain, what it is a complementary filter, benefits and internal structure. We will include a short code in the Clanguage for data processing from the output.

Keywords: stabilize, accelerometer, gyroscope, complementary filter, DSP

\section{Introduction}

We can find stabilization systems in many electrical devices where they are used to control the vehicle's direction or to stabilize the platform. For example, the force that acts on a platform deflects this platform from balance. However, this is undesirable. Therefore, we try to keep the platform in the surface using a proposed stabilisation system. One way is to use tilt sensors and design a suitable hardware. It is necessary to know the physical model of the platform and to determine the correct system parameter.

\section{Theoretical knowledge of stabilization systems}

To stabilize a single or two-wheeled vehicle, it is necessary to know the angle of inclination of the platform against the horizontal axis and also what motion of the vehicle is carried out. It can be detected by several ways, such as with the mercury tube, which was used in the tilt sensor technologies in motor vehicles. Today, accelerometers and gyroscopes are small, accurate and energy-saving. They provide analogue or digital output.

\subsection{Accelerometer}

Accelerometer is an electromechanical device that measures the acceleration force. This force may be static, such as the force of gravity or dynamic by motion or vibration of the accelerometer. There are many types of accelerometers. The most sensors are based on piezoelectric devices using crystals, but they are large. Therefore, MEMS (micro electro-mechanical system) are used most often. They are able measure power in three axes.
A typical MEMS accelerometer consists of a movable mass with attached membranes through a mechanical suspension to the frame as shown in Figure 1 [1].

Movable plates and solid external boards represent capacitors. The deflection of the movable plate towards the center is measured by the difference in capacity. The capacity of the free space (air) between the movable plate and the two stationary outer plates $\mathrm{x}_{1}$ and $\mathrm{x}_{2}$ is a function corresponding to the displacement $\mathrm{x}_{1}$ and $\mathrm{x}_{2}$ :

$$
\begin{aligned}
& C_{1}=\in_{A} \frac{1}{x_{1}}=\in_{A} \frac{1}{d+x}=C_{0}-\Delta C \\
& C_{2}=\in_{A} \frac{1}{x_{2}}=\in_{A} \frac{1}{d-x}=C_{0}+\Delta C
\end{aligned}
$$

where $C_{A}$ is the permittivity of the separating material and $d$ is the distance between electrodes.

If acceleration is zero, the capacity $\mathrm{C}_{1}$ and $\mathrm{C}_{2}$ are the same, because $x_{1}=x_{2}$. If $x \neq 0$, the capacity will be:

$$
C_{2}-C_{1}=2 \Delta C=2 \in \frac{x}{d^{2}-x^{2}}
$$

By adjusting we get a nonlinear algebraic equation:

$$
\Delta C x^{2}+\in{ }_{A} x-\Delta C d^{2}=0
$$

We can simplify the equation. But for small shifts $\Delta \mathrm{CX}^{2}$ is obscure, so $\Delta \mathrm{Cx}^{2}$ we can ignore. Then:

$$
x:=\frac{d^{2}}{\in_{A}} \Delta C=d \frac{\Delta C}{C_{0}}
$$

Capacitors $C_{1}$ and $C_{2}$ represent a simple voltage prescaler.

$$
\left(V_{x}+V_{0}\right) C_{1}+\left(V_{x}+V_{0}\right) C_{2}=0
$$

\footnotetext{
* ${ }^{1}$ Patrik Beno, ${ }^{1}$ Miroslav Gutten, ${ }^{1}$ Milan Simko, ${ }^{2}$ Jozef Sedo

'Department of Measurements and Applied Electrical Engineering, Faculty of Electrical Engineering and Information Technology, University of Zilina, Slovakia

${ }^{2}$ Department of Mechatronics and Electronics, Faculty of Electrical Engineering and Information Technology, University of Zilina, Slovakia E-mail: gutten@fel.uniza.sk
} 


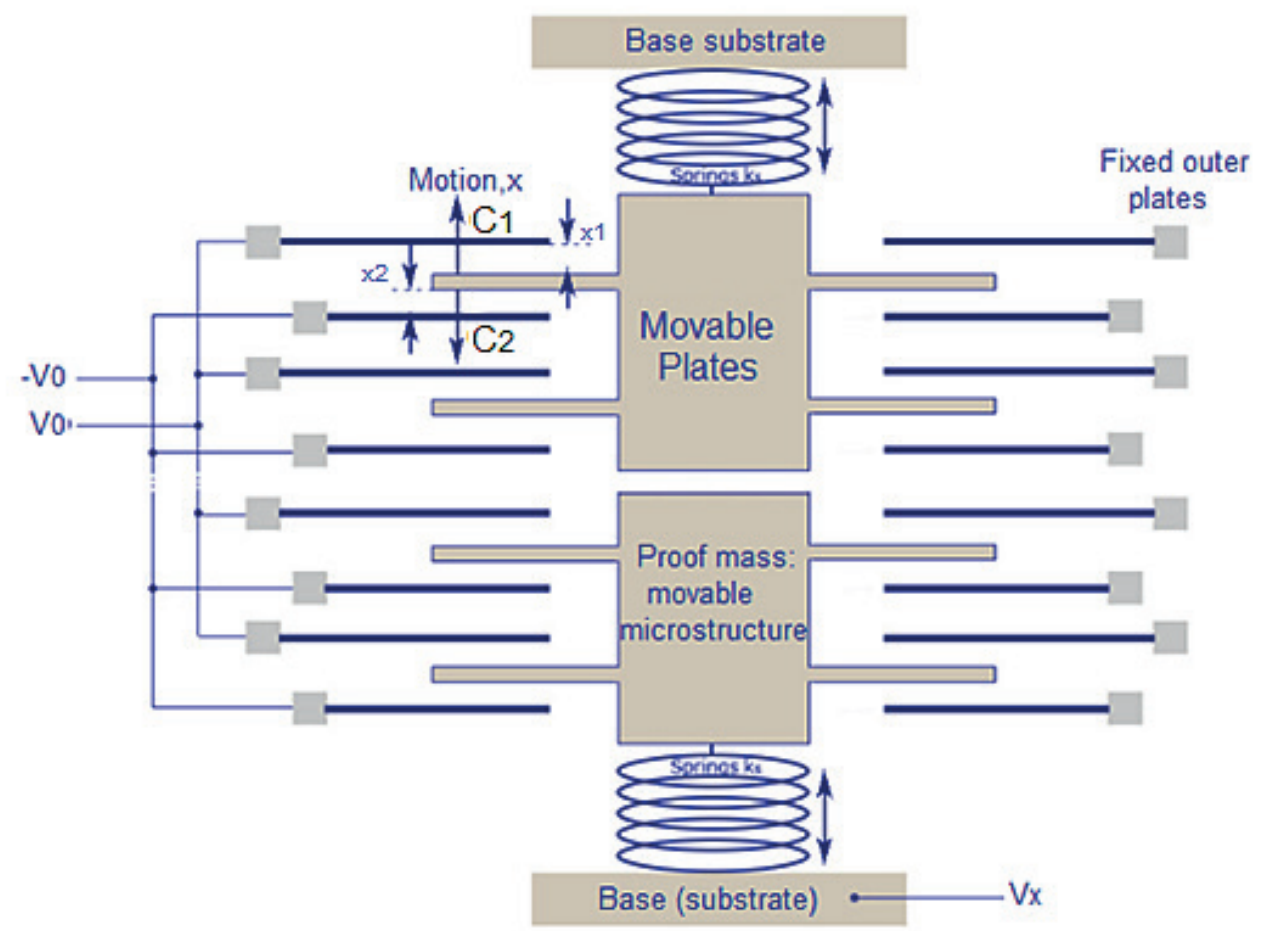

Figure 1 Structure of accelerometer

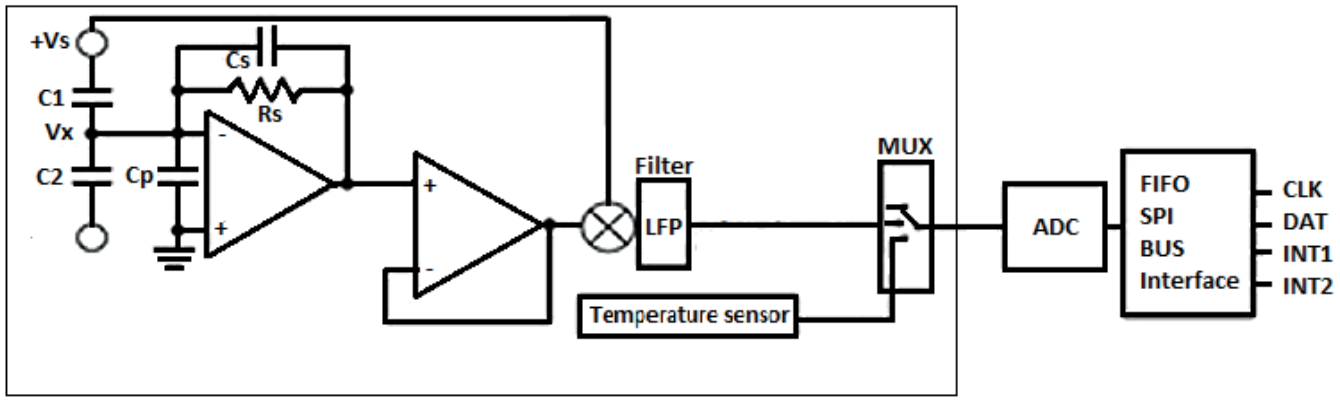

Figure 2 Accelerometer measurement circuit

Then we get the output voltage $\mathrm{V}_{\mathrm{x}}$ :

$V_{x}=V_{0} \frac{C_{2}-C_{1}}{C_{2}+C_{1}}=\frac{x}{d} V_{0}$

The output signal $V_{\mathrm{x}}$ cannot be used without filtering because the signal is noisy. Therefore, it is necessary to use a circuit consisting of an integrated amplifier, a separator, a low pass filter and a multiplexer. It is also necessary to convert the signal into digital form to get the data from the accelerometer and gyroscope via the I2C bus. This connection is shown in Figure 2.

\subsection{Gyroscope and integration}

The classic gyroscope is defined as a symmetrical rotating object around one axis. These are very large for use in electronics. Now, we are using new types of piezoelectric gyroscopes or laser ray gyroscopes, which works on the principle of laser ray deflection. Vibrating gyroscopes MEMS work on the principle of mass resonance inside the structure. The direction which we will consider the motion of a body is marked x, see Figure 3 [2].

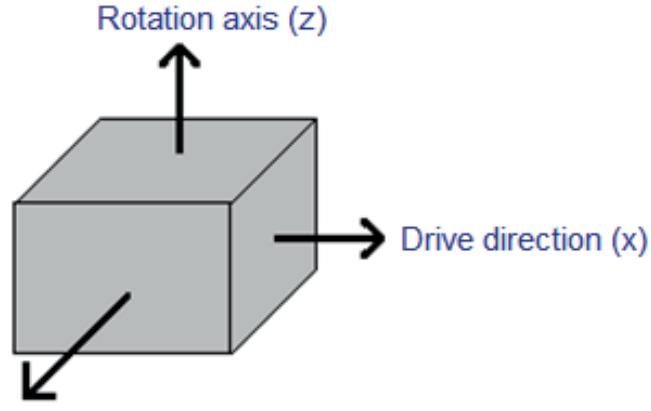

Sense direction (y)

Figure 3 Gyroscope measurement axis

The output signal of the gyroscope is digital and is dependent on the system rotation speed in degree per second $(\% / s)$. MEMS gyroscopes are radically less sensitive to the applied acceleration than accelerometers [3]. To obtain a tilt value at time $t_{1}$, the output signal must be integrated in time according to (Figure 4):

$\varphi=\int_{0}^{t_{1}} \omega(t) d t+\varphi(0)$ 


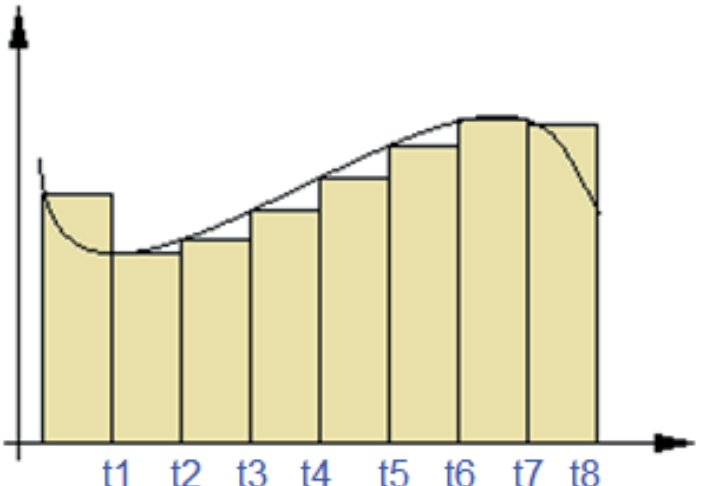

a)

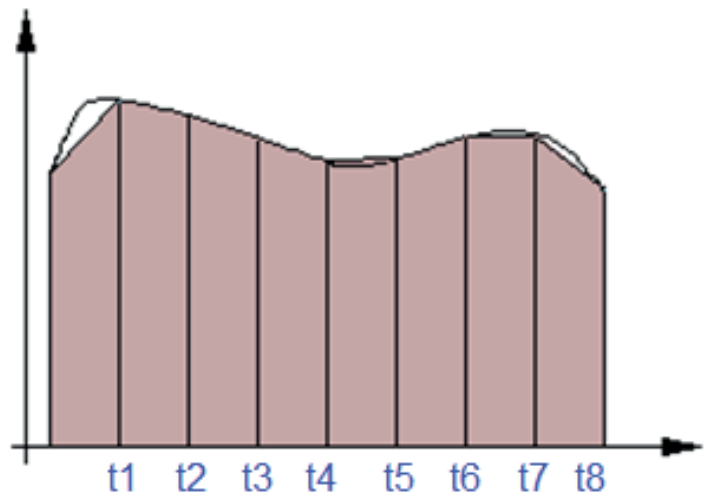

b)

Figure 4 (a) Rectangular integration, (b) Trapezoidal integration

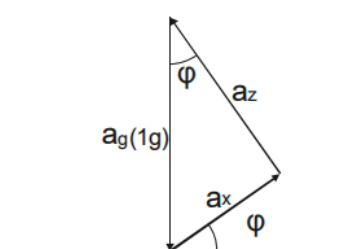

Figure 5 Trigonometry of angle measurement by accelerometer

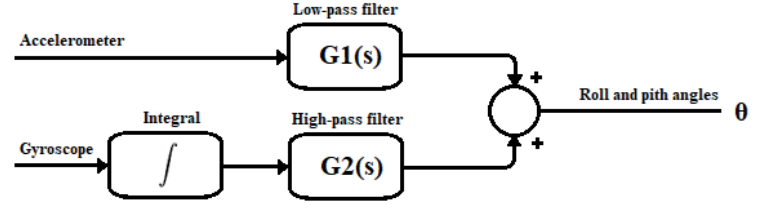

Figure 6 Complementary filters with constant gain

\subsection{Accelerometer and angle measurement system}

MEMS accelerometers can measure static and dynamic acceleration. If we have a system which we incline around the $\mathrm{Y}$ axis, we can observe the change of the measured value of the acceleration $\alpha_{\mathrm{x}}$ a $\alpha_{\mathrm{z}}$ depending on gravity acceleration $\alpha_{\mathrm{g}}$ [4].

This results in relations for angles (Figure 5):

$\alpha_{\mathrm{x}}=\alpha_{\mathrm{g}} \sin \varphi$

$\alpha_{\mathrm{z}}=\alpha_{\mathrm{g}} \cos \varphi$

Then we can calculate the resulting angle $\varphi$ by gravity acceleration $\alpha_{\mathrm{x}}$ and $\alpha_{\mathrm{z}}$ with expression [5]:

$\varphi=\operatorname{atan}\left(\frac{\alpha_{x}}{\alpha_{z}}\right)$

By using the 3-axis accelerometer, we obtain tilt angles in the NED (North-East-Down) coordinate system. It is also necessary to define the angle of elevation, which $\mathrm{i}$ is marked $\theta$ and angle of pitch is marked as $\Phi[6]$ :

$$
\begin{aligned}
& \theta=\operatorname{atan}\left(\frac{\alpha_{x}}{\alpha_{z}}\right) \\
& \Phi=\operatorname{atan}\left(\frac{\alpha_{x}}{\sqrt{\alpha_{y}^{2}+\alpha_{x}^{2}}}\right)
\end{aligned}
$$

\section{Design of complementary filter with constant gain}

The MPU6050 sensor with an integrated accelerometer and gyroscope was used for the tilt sensing. A complementary filter has been designed to evaluate the data, which includes all signal processing requirements for control. [7]. The complementary filter is one of the filtering techniques in the frequency domain. Two or more variables are entering to the complementary filter. Only part of the spectrum is used from each sensor, and all sensors cover the frequency spectrum [8]. The proposed block diagram of the filter is shown in Figure 6, where the input parameters are the real accelerometer values and the gyroscope values must be first integrated. [9].

This applies:

$G 1(s)+G 2(s)=1$

where $G 1$ (s) is a high pass filter and $G 2(\mathrm{~s})$ is the low pass filter.

The proposed calculation in $\mathrm{C}$ code is shown below. The „alpha“ is variable, in this case 0.9 .

Then apply:

angle $=(1-$ alpha $) *($ gyro $)+($ alpha $) *($ acc $)$

where:

- $\quad$ angle $=$ output angle from filter

- gyro $=$ measured angle from the gyroscope

- $\quad$ acc $=$ measured angle from the accelerometer

This filter has been implemented into the control unit for the BLDC motor of a one-wheeled vehicle with the NXP MC56F8006 processor. MPU6050 sensor is connected via connector SV7, see Figure 7.

The processor every $10 \mathrm{~ms}$ takes a value from the accelerometer and gyroscope and computes the result value of the angle, which then enters to the PI controller. Then it discards the difference between the actual and the desired value. [10]. The constants of the PI controller are determined by the Ziegler Nicholson 


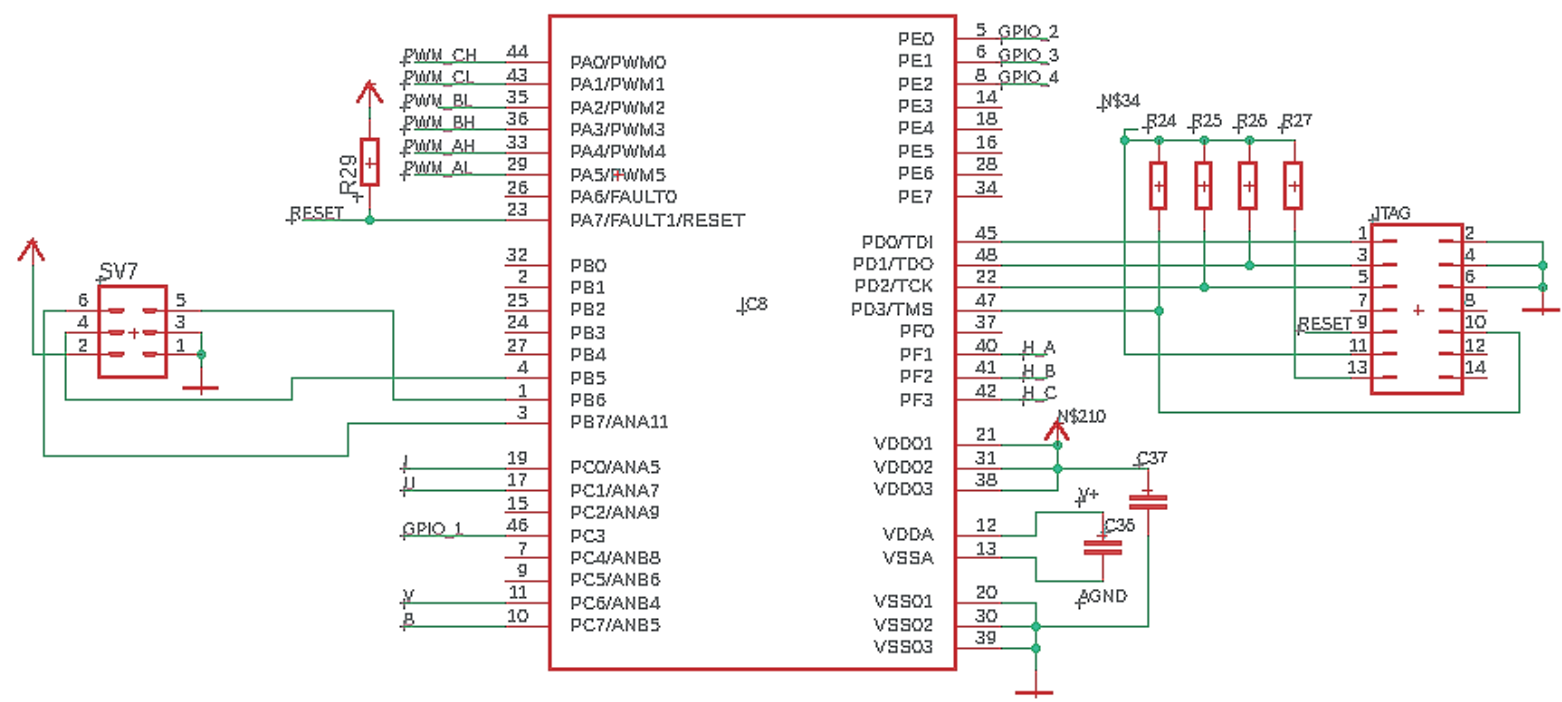

Figure 7 Controlling processor

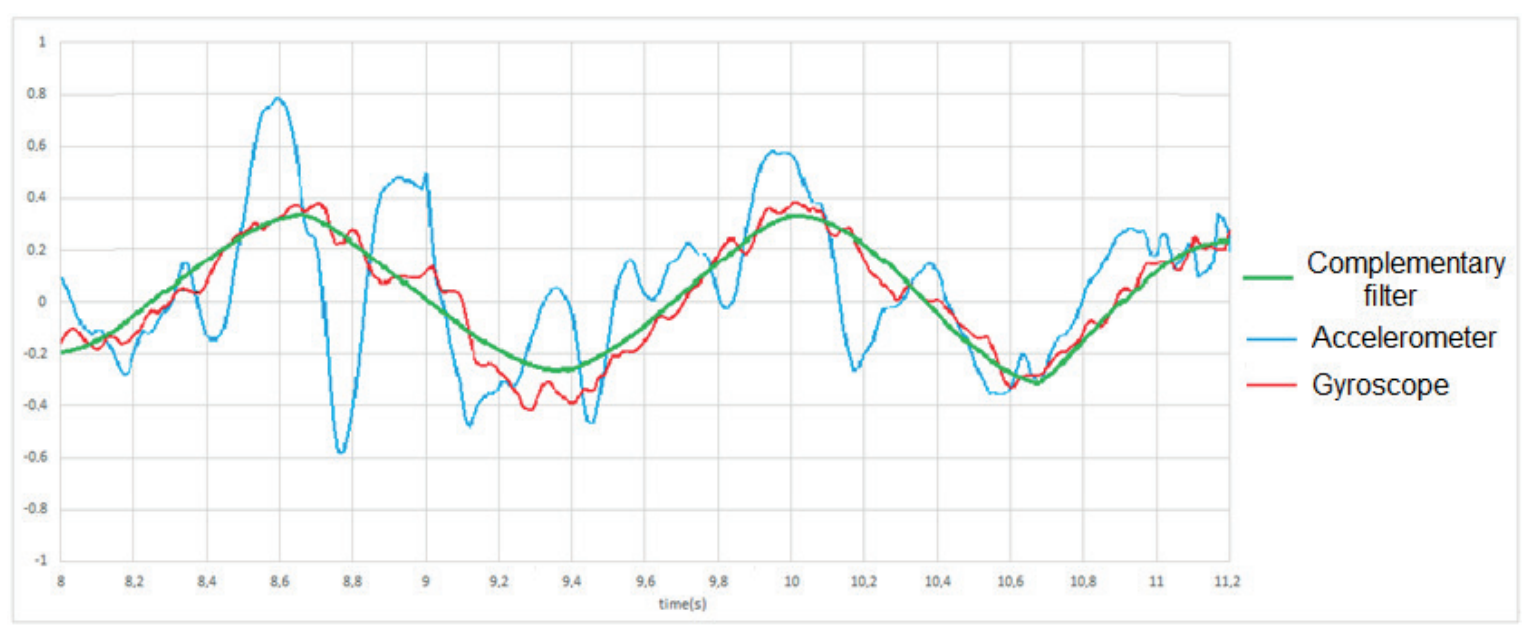

Figure 8 Output of accelerometer, gyroscope and complementary filter

method. This value is entered into other calculations and PD controller for electric motor drive.

We can see output values of the accelerometer, a gyroscope and a complementary filter in Figure 8.

\section{Conclusion}

We see a lot of differences between the accelerometer and gyroscope, but by their use we get sufficient inclination measuring system in one axis. The system will then be modified by adding a tilt measurement of the two remaining axes for parameter improvement.
The designed complementary filter is suitable for controlling the one-wheeled or two-wheeled platform. We can see the graph, where the filter softens the accelerometer and the gyroscope values and the resulting course is usable for controlling the converter.

\section{Acknowledgements}

This article is the result of a project implementation: Modern methods of teaching of control and diagnostic systems of engine vehicles, ITMS code 26110230107, supported by the Operational Programme Educational. 


\section{References}

[1] WAGNER F., SCHMUKI R., WAGNER T., WOLSTONHOLME P. Modeling software with finite state machines: a practical approach. Boca Raton, FL: Taylor \& Francis Group, 2006. ISBN 978-0-8493-8086-0.

[2] BURG A., MERUANI A., SANDHEINRICH B., WICKMANN M. Mems gyroscopes and their applications. A study of the advancements in the form, function, and use of mems gyroscopes. Final Report. Northwestern University, Mechanical Engineering Department, ME 381 - Introduction to MENS, prof. H. D. Espinosa, 2002.

[3] ELLIOTT, K. S., GUPTA, P., REED K. B., RODRIGUEZ R. C. Micromachined vibrating gyroscopes: design and fabrication. Final Report. Northwestern University, Mechanical Engineering Department, ME 381 - Introduction to MENS, prof. H. D. Espinosa, 2002.

[4] RODINA, J. Application of inertial sensors for the control of mobile robotic platforms. Autoreport of dissertation work. 2014.

[5] MCGILVRAY S., Self-erecting inverted pendulum: swing up and stabilization control. Central Canada Council for the IEEE Student Paper Contest, 2002.

[6] ANDREJASIC M. Mems accelerometers. Seminar. University of Ljubljana, Faculty for mathematics and physics, Department of physics, 2008.

[7] ELLIOTT-LABORATORIES. The anschutz gyro-compass and gyroscope engineering. Kiel, Germany: Wexford College Press, 2003, p. 7-24. ISBN 978-1-929148-12-7.

[8] MUCHERU G. Operation of gyro sensor and 3-axis accelerometer. Thesis. Helsinki Metropolia University of Applied Sciences, 2014.

[9] SEDO, J., KASCAK, S. Design of output LCL filter and control of single-phase inverter for grid-connected system. Electrical Engineering [online]. 2017, 99(4), p. 1217-1232. ISSN 0948-7921, eISSN 1432-0487. Available from: https://doi.org/10.1007/s00202-017-0617-0

[10] DOBRUCKY B. Discrete control of power electronic systems. Zilina: EDIS - Publishing House of the University of Zilina, 2003. 